\title{
Dientes posteriores tratados endodónticamente: Alternativas para su rehabilitación basadas en evidencia científica. Revisión de la literatura
}

\author{
Endodontically treated posterior teeth: Alternatives for their rehabilitation based on scientific \\ evidence. Literature review \\ Dentes posteriores tratados endodonticamente: Alternativas para sua reabilitação com base em \\ evidências científicas. Revisão de literatura
}

Recibido: 08/03/2021 | Revisado: 10/03/2021 | Acepto: 12/03/2021 | Publicado: 18/03/2021

Amy Paola Aguirre Segarra

ORCID: https://orcid.org/0000-0002-9939-2264 Universidad de Cuenca, Equador

E-mail: amy.aguirre@ucuenca.edu.ec

Tannya Cristina Rodríguez León

ORCID: https://orcid.org/0000-0002-5102-8826 Universidad de Cuenca, Equador

E-mail: tannya.rodriguez@ucuenca.edu.ec

Yulissa Raquel Abad Salinas

ORCID: https://orcid.org/0000-0002-5450-7603 Universidad de Cuenca, Equador

E-mail: yulissa.abad@ucuenca.edu.ec

\begin{abstract}
Resumen
La restauración definitiva de un diente tratado endodónticamente (DTE) es fundamental para prevenir una posible reinfección periapical, lo que determina su pronóstico a corto y largo plazo. Este trabajo tiene como objetivo determinar la tasa de sobrevida y qué tipo de restauración está indicada en dientes posteriores tratados endodónticamente de acuerdo a la pérdida de estructura dentaria. Para ello, se realizó una búsqueda en las bases digitales Cochrane, Scopus y Pubmed acorde a criterios de inclusión y exclusión; y una búsqueda manual en Google Scholar. Como resultado se obtuvieron 34 artículos para la lectura de texto completo, se tomó dos clasificaciones que permitieron a los revisores unificar criterios y proponer una nueva clasificación que facilitará a los lectores elegir la forma de restauración ideal en los dientes posteriores. Para aquellas piezas dentarias con perdida mínima de estructura coronaria, el tratamiento de elección son restauraciones de composite (91.9\%-100\% de éxito); en piezas con pérdida moderada de tejido dentario, se recomienda la utilización de restauraciones indirectas de cobertura parcial o total (92.4\%-98.9\%) con el uso o no de un poste de fibra de vidrio y, por último, en piezas con gran pérdida de remanente se optaría por técnicas alternativas que permitan preservar tejido dentario. Es preciso conocer las opciones existentes al momento de escoger un tipo de restauración conforme a los requerimientos de la pieza dentaria, considerando ser lo más conservador posible para no debilitar aún más su estructura.
\end{abstract}

Palabras clave: Restauración; Diente tratado endodónticamente; Diente no vital; Diente posterior.

\begin{abstract}
The definitive restauration of an endodontically treated tooth (ETT) is essential to prevent a possible periapical reinfection, which determines its short- and long-term prognosis. The aim of this paper is to determine the survival rate and the type of restoration that should be prescribed in posterior teeth according to the loss of tooth structure. In order to be able to achieve this, a search was conducted using digital databases such as: Cochrane, Scopus and PubMed using certain inclusion and exclusion criteria; as well as a manual search performed using Google Scholar. As a result, thirty-four articles were obtained for full reading, from which two classifications emerged, which allowed the reviewers to join criteria and propose a new classification which will make life easier for the reader at the time of choosing the ideal restoration. For those teeth with minimal loss of structure, the treatment of choice is composite restorations (91.9\% to $100 \%$ rate of success); in teeth with a moderate loss of dental structure the ideal treatment was the use of indirect restorations of partial or total coverage ( $92.4 \%$ to 98.95 rate of success) alongside a fiber post if deemed necessary, finally, in teeth with a great loss of dental structure alternative techniques should be chosen in order to preserve as much dental tissue as possible. It is necessary to recognize the available options at the time of choosing a type of restoration according to the requirements of the tooth, always considering to be as conservative as possible so as not to further weaken its structure.
\end{abstract}

Keywords: Restoration; Endodontically treated teeth; Non-vital teeth; Posterior teeth.

\section{Resumo}

A restauração definitiva de um dente tratado endodonticamente (DTE) é essencial para prevenir uma reinfecção periapical, o que determina o seu prognóstico de prazo curto ou longo. O objetivo deste artigo é para determinar a taxa 
de sobrevivência e o tipo de restauração que deve ser prescrito nos dentes posteriores de acordo com a perda de estrutura dentária. Para conseguir isso, uma pesquisa foi realizada usando bancos de dados digitais tais como: Cochrane, Scopus e PubMed usando certos critérios de inclusão e exclusão; assim como uma pesquisa manual realizada usando Google Scholar. Como resultado, trinta e quatro artigos foram obtidos para leitura completa, de onde surgiram duas classificações que permitiu que os revisores juntassem os critérios e propor uma nova classificação que fará a vida dos leitores mais fácil na hora de escolher a restauração ideal. Para os dentes com a perda mínima de estrutura, o tratamento de escolha são as restaurações compostas (de $91.9 \%$ à 100\% taxa de sucesso); nos dentes com a perda moderada de estrutura dentária o tratamento ideal que foi usado foi restauração indireta de cobertura parcial ou total (de 92.4 à $98.95 \%$ taxa de sucesso) ao lado de uma coluna de fibra se necessário, finalmente, nos dentes com uma maior perda de estrutura dentária, técnicas alternativas devem ser escolhidos a fim de preservar o máximo de tecido dentário possível. É necessário reconhecer as opções disponíveis na hora de escolher um tipo de restauração de acordo com as necessidades do dente, considerando sempre ser o mais conservador possível para não enfraquecer ainda mais sua estrutura.

Palavras-chave: Restauração; Dentes tratados endodonticamente; Dentes não vitais; Dentes posteriores.

\section{Introducción}

El éxito de un diente tratado endodónticamente (DTE) está estrechamente relacionado con el correcto sellado coronal, el cual protegerá al diente de la microfiltración bacteriana y una posible reinfección de los tejidos periapicales; por lo que seleccionar una adecuada restauración es un verdadero reto, ya que establece el pronóstico a largo plazo del DTE (Monardes et al., 2016; Zarow et al., 2018).

La planificación del tratamiento restaurador definitivo de un DTE incluirá una evaluación exhaustiva e integral, la cual deberá ser factible para el odontólogo y accesible para el paciente. Existen diversos factores a considerar al momento de elegir el tipo de restauración, los más importantes son: tejido coronal remanente, tejido radicular residual, valor del diente comprometido en el arco, posición y función de la pieza en la arcada, entre otros (Zarow et al., 2018). De todos los mencionados, el primero fue seleccionado como referencia para realizar esta investigación, pues comúnmente en la práctica clínica los DTE suelen presentar gran destrucción de sus paredes a nivel coronario, recalcando que, algunas piezas dentarias debieron estar sometidas a un proceso de caries que culminó con la afectación de la pulpa.

Con el transcurso del tiempo han surgido varias clasificaciones en torno a la cantidad de estructura dental residual y el tipo de restauración idónea para dicho caso. La primera y más utilizada es citada por Peroz et al., publicada en el año 2005, describe de forma detallada la cantidad de tejido coronal residual puesto que la extensión de la destrucción no se puede evaluar métricamente (Peroz et al., 2005); y la segunda, más actual, publicada en el año 2017 por Zarow et al; se creó con el propósito de ayudar al clínico a escoger un plan de tratamiento para restaurar un DTE según la conformación del muñón (Zarow et al., 2018).

Por lo tanto, el objetivo de esta revisión es determinar la tasa de sobrevida y qué tipo de restauración está indicada en dientes posteriores tratados endodónticamente de acuerdo a la pérdida de su estructura dentaria.

\section{Metodología}

Para la presente revisión se empleó un componente metodológico cualitativo, de carácter descriptivo, para ello se realizó una búsqueda en las bases digitales como son Cochrane, Scopus y Pubmed en el idioma inglés y que fuesen publicados en los últimos 5 años, teniendo como finalidad responder a la siguiente pregunta: “¿Qué tipo de restauraciones presentan mayor éxito en los dientes posteriores tratados endodónticamente de acuerdo a la pérdida de su estructura dentaria?”, para lo cual se emplearon las siguientes palabras claves: restoration; endodontically treated teeth; non vital teeth; posterior teeth, se ejecutó una búsqueda manual para obtener información complementaria, dicha indagación se realizó en Google Scholar. 


\section{Tipos de estudios y criterios de selección}

- Criterios de inclusión: para esta revisión se consideraron estudios ECA, revisiones sistemáticas y con metaanálisis, se valoró el número de paredes coronales restantes después del tratamiento endodóntico, restauraciones directas e indirectas, sobrevida y fracaso de las restauraciones.

- Criterios de exclusión: Los estudios se excluyeron si: no pertenecían a los términos de búsqueda descritos en los criterios de inclusión, estudios in vitro, dientes temporarios y dientes anteriores de la dentición permanente.

\section{Resultados}

La búsqueda electrónica identificó 2560 artículos. Dos revisores examinaron de forma independiente los resultados, se aplicaron los criterios de inclusión y exclusión; y se eliminaron los duplicados, obteniendo 19 estudios. Tras la revisión de títulos y resúmenes, se seleccionaron 12 para el análisis de texto completo. La búsqueda manual arrojó 22 más, generando un total de 34 artículos que sustentan esta investigación.

\section{Discusión}

Las clasificaciones de Peroz y Zarow, fueron el fundamento para dar lugar a una nueva guía mediante la unificación de criterios, con el objeto de facilitar al lector la elección de un tipo u otro de restauración en base a la tasa de supervivencia reportada. Dichas clasificaciones se detallan a continuación:

\subsection{Clasificación de Peroz (2005)}

Clase I: La pieza dentaria tiene sus 4 paredes remanentes intactas, por lo que se considera cualquier tipo de restauración definitiva, generalmente composites.

Clase II y III: La pieza dentaria posee 2 o 3 paredes residuales, no requiere la colocación de un poste ya que el tejido proporciona suficiente estabilidad para el uso de otros métodos que emplean sistemas adhesivos.

Clase IV: La pieza dentaria tiene 1 pared remanente, implica el uso de postes. Por razones estéticas, se prefieren los postes no metálicos para el tratamiento de dientes anteriores y posteriores. A pesar de ello, los postes metálicos como los no metálicos son opciones de tratamiento aceptables.

Clase V: No existen paredes remanentes, por lo que es vital colocar un poste con el fin de propiciar resistencia al muñón. Es imperioso obtener un efecto férula para brindar mayor resistencia a la fractura; en caso de que la destrucción extensa de la corona haga imposible una férula suficiente, se puede llevar a cabo un alargamiento quirúrgico de la corona (Peroz et al., 2005).

\subsection{Clasificación de Zarow (2017)}

Clase 0: La pieza dentaria cuenta con todas sus paredes, proporcionando retención mecánica; por lo que el tratamiento de elección es una restauración directa.

Clase 1: Está indicada la colocación de un poste en dientes anteriores y premolares con dos o menos paredes; en los molares no es necesario, excepto en casos de tejido coronal insuficiente. 
Clase 2: Cuando se quiere recuperar el efecto férula, la decisión se basa en la posición del diente en el arco, en el caso de los molares se puede realizar alargamiento quirúrgico de la corona y en dientes anteriores y premolares, extrusión ortodóntica; previo al tratamiento restaurador.

Clase 3: Piezas con mínima estructura residual y sin la posibilidad de generar efecto férula a través de procesos ortodónticos o periodontales, y el paciente aún desea conservar la pieza dentaria; se podría optar por la colocación de un poste de oro fundido o de aleaciones de plata-paladio. (Zarow et al., 2018).

La restauración final de un DTE tiene como objetivo mantener la estética y función del diente en cuestión (Victoria, 2019), además propicia un sellado coronal apto, el cual influye directamente en la calidad del tratamiento endodóntico y con ello, el pronóstico de la pieza dentaria (Vallejo Labrada \& Maya Cerón, 2015).

Atlas et al., (2019) evaluaron en su estudio el éxito de DTE con una apropiada restauración coronal, en el que el 90\% de los dientes que presentaban un apropiado sellado, se hallaban libres de infecciones a nivel apical; pero, aquellos que tenían alteraciones en la integridad marginal, tuvieron un éxito del 44\%. Por tanto, si la restauración definitiva es de mala calidad o si el proceso de obturación se completa de forma deficiente, disminuye en gran medida el éxito esperado tanto a corto como a largo plazo.

La restauración post tratamiento de conducto radicular se ha transformado en un tema controversial en las últimas décadas debido a la diversidad terapéutica y materiales existentes. La literatura sugiere dos alternativas: las restauraciones directas e indirectas, considerando dentro del primer grupo, las restauraciones de composite y en el segundo grupo, las de cobertura parcial y total (Afrashtehfar et al., 2017).

Algunos años atrás, la restauración indirecta era más común y consistía en la colocación de un poste y una corona de cobertura total; sin embargo, se encontraron ciertas desventajas tales como: desgaste excesivo de tejido dentario, mayor tiempo de trabajo, costo superior en relación con las restauraciones directas (Afrashtehfar et al., 2017; Govare \& Contrepois, 2019). Por consiguiente, las formas de restauración pueden variar acorde a las condiciones de la pieza dentaria a tratar.

El objetivo principal de cualquier tratamiento dental es preservar la mayor cantidad de tejido duro, especialmente en casos de DTE que han perdido gran cantidad de tejido coronal, es crucial la conservación del efecto férula.

\section{Importancia del efecto férula en la restauración de DTE}

Una férula es un collar de dentina circunferencial de aproximadamente $2 \mathrm{~mm}$ de altura (Sarkis-Onofre et al., 2017), que genera un adecuado comportamiento biomecánico brindando suficiente estabilidad y resistencia a la fractura a la pieza dentaria (Magne \& Spreafico, 2012).

Algunos autores consideran que una férula de $1.5 \mathrm{~mm}$ a $2 \mathrm{~mm}$ de altura y de $1 \mathrm{~mm}$ de espesor como se observa en la Figura 1, es esencial para incrementar en un 5\% la probabilidad de supervivencia a mediano (5 años) y largo plazo (17 años) y lograr un valor funcional de soporte (Naumann et al., 2017). 
Figura 1: Efecto férula.

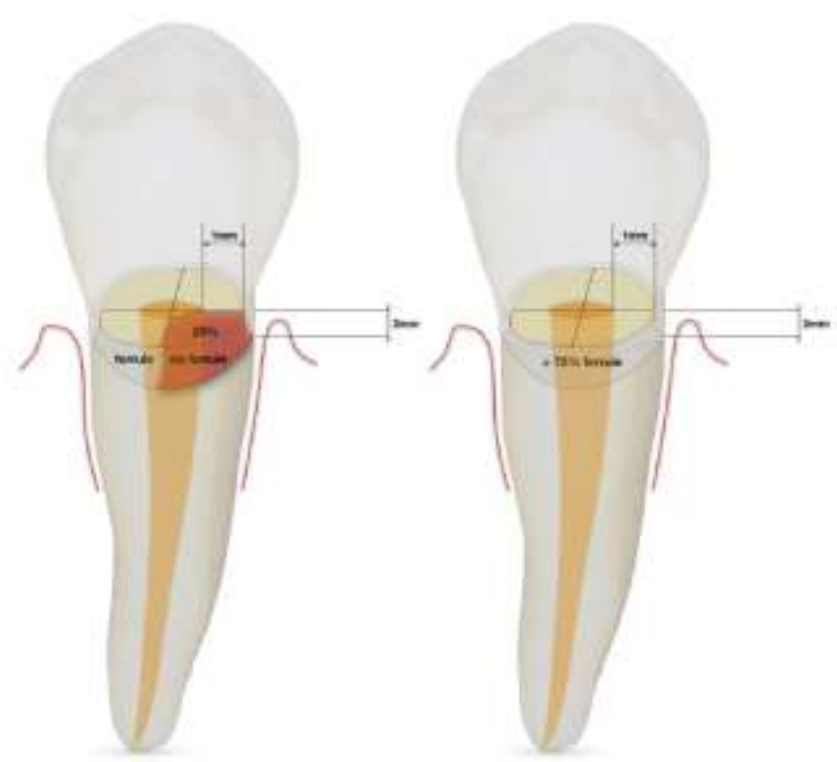

Fuente: Naumann et al. (2017).

Naumann et al., (2017) llevaron a cabo un seguimiento de 5 años a piezas dentarias restauradas con efecto férula, las cuales tuvieron una tasa de supervivencia mayor (98\%) que aquellas sin este (93\%).

$\mathrm{Su}$ presencia confiere un efecto protector durante la inserción de un poste, pues otorga resistencia a las fuerzas funcionales, las fuerzas laterales y al efecto de cuña de los postes metálicos. Asimismo, impide la exposición directa del cemento y consecuentemente, una reducción de fallas como desprendimientos de la corona o la posibilidad de microfiltraciones (Batista et al., 2020).

\subsection{Nueva clasificación propuesta}

\subsubsection{DTE con 4 paredes remanentes:}

Corresponde a la Clase I (Peroz et al., 2005) y Clase 0 (Zarow et al., 2018), se indican restauraciones directas cuando la cavidad es pequeña, por ejemplo si la pérdida de estructura dentaria se atribuye a la conformación de la cavidad de acceso; su tasa de supervivencia es del 100\% a corto plazo y del 91.9\% a largo plazo (Cagidiaco et al., 2007; Dammaschke et al., 2013; Mannocci et al., 2009; Suksaphar et al., 2018).

En caso de cavidades amplias pero que conservan sus cuatro paredes, se optaría por restauraciones indirectas; las cuales requieren paredes con un grosor mínimo de $3 \mathrm{~mm}$; así pues, el clínico deberá decidir si conservar el tejido dentario y modificar su morfología con un material adecuado, a esta técnica se la conoce como build up (Figura 2), o eliminarlo y ejecutar un recubrimiento cuspídeo cuando el grosor de las paredes no sea uniforme o sea menor al requerido, con el objetivo de prevenir una posible fractura coronal, reducir la posibilidad de crear fisuras en el esmalte y una deficiencia a nivel marginal (Ferraris, 2017). 
Figura 2: A. Radiografía final de un molar tratado endodónticamente. B. Remoción de material provisional para análisis de estructura dentaria y elección de tratamiento restaurador C. Colocación de resina bulk para rellenar la cavidad y colocar restauración indirecta tipo onlay.
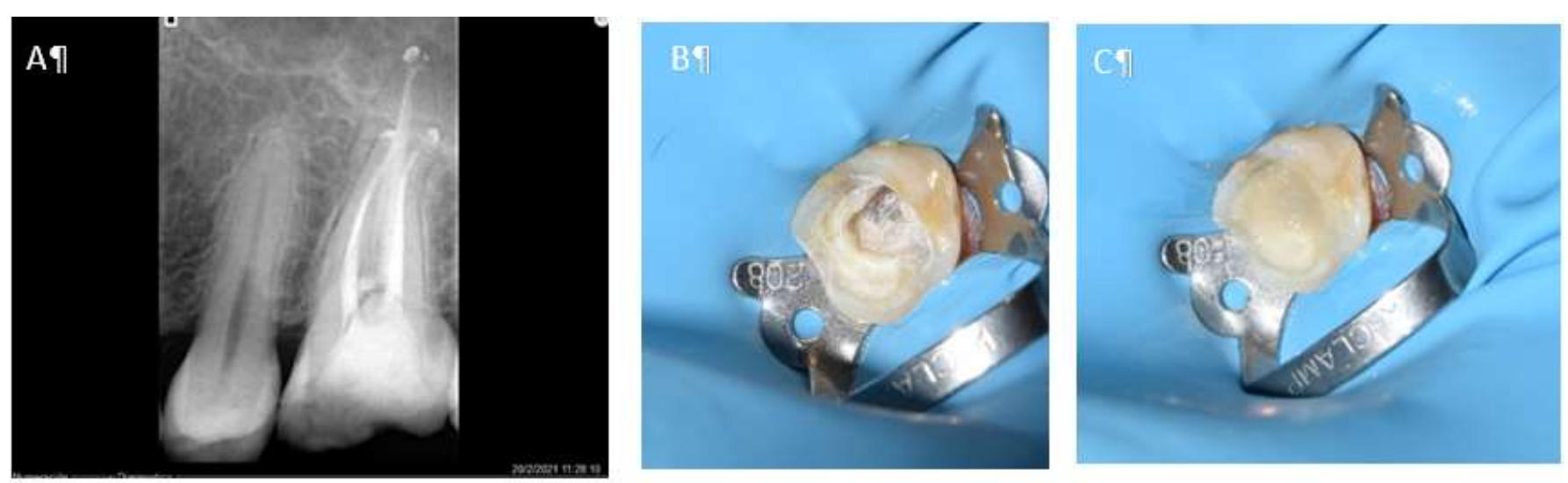

Fuente: Od. Esp. Yulissa Abad / Od. Esp. Lorena Carpio.

\subsubsection{DTE con 3 paredes remanentes}

Existen dos alternativas para las clases II y III de Peroz (Peroz et al., 2005). La primera consiste en restauraciones de composite, considerando una tasa de supervivencia del 85,5\%; no obstante, es fundamental recalcar que existe una mayor incidencia a la fractura (Jirathanyanatt et al., 2019); la segunda hace referencia a restauraciones indirectas de cobertura parcial: inlay, onlay y overlay.

Ferrari et al., (2019) efectuaron un seguimiento de 3 años a las restauraciones de dientes posteriores, en los cuales se empleó o no postes de fibra de vidrio e incrustaciones; cuyos resultados se establecen como satisfactorios al exhibir una tasa de supervivencia del 93,3\% para premolares y $100 \%$ para molares. Shu et al., (2018) demostraron que las restauraciones indirectas tienen éxito en un rango de 5 y hasta 10 años en comparación con restauraciones de composite.

Estudios a largo plazo evidencian que DTE que han sido restaurados con inlays u onlays de cerámica tienen un rango de éxito del $98.9 \%$ a los 5 años, del 92.4\% a 96.8\% después de 10 años y del 89.6\% a 92.4\% luego de 12 años; en contraste a las restauraciones indirectas de composite que muestran una tasa de sobrevida del 80\% al 88\% a los 12 años (Beier et al., 2012; Bresser et al., 2019).

\subsubsection{DTE con 1 o 2 paredes remanentes}

En aquellas piezas dentarias que han perdido el $50 \%$ de estructura coronaria, concerniente a la Clase IV de Peroz y Clase 1 de Zarrow, se debe tener en cuenta si existe o no efecto férula.

En presencia de ella, las piezas dentarias que conservan dos paredes pueden ser reconstruidas con composite sin necesidad de un poste para colocar una corona simple (Naumann et al., 2017). Dammaschke et al., (2013) en su estudio presentaron piezas con pérdida de 1 a 5 paredes en las cuales se trató con coronas de cobertura total, se reportó una tasa de supervivencia de $94 \%$. Un estudio a corto plazo mostró que una pared cavitaria remanente puede sustituir el efecto del poste (Bitter et al., 2009; Naumann et al., 2017).

En ausencia de dicho efecto, se indica la colocación de poste y la reconstrucción del muñón con composite para su posterior restauración con una corona de recubrimiento total, lo cual evidencia una tasa de supervivencia del 95,1\% (Suksaphar et al., 2018). 


\section{Influencia del poste en la restauración de un DTE}

Por mucho tiempo, se ha creído que el papel del poste es reforzar la estructura radicular, actualmente se conoce que no desempeña realmente una función debido a su posición en el centro del diente; sino crea una retención adicional para la reconstrucción del muñón (Naumann et al., 2018).

Antiguamente se empleaban postes metálicos (Figura 3A), hoy en día se encuentran en desuso debido a numerosas desventajas, entre ellas: su alto módulo de elasticidad (220 GPa) (Sorrentino et al., 2016), la posible corrosión de las aleaciones metálicas y su combinación con diversos materiales con los que las coronas o incrustaciones están elaboradas, mismos que contribuyen a la fractura de la pieza dentaria; además, por su opacidad, interfieren en el paso de la luz, disminuyendo la transparencia de la encía, visualizándose una zona oscura a nivel del festón gingival y dando un aspecto poco o nada estético (Aguero et al., 2017).

Figura 3: Postes intrarradiculares. A. Poste metálico. B. Poste de fibra de vidrio.
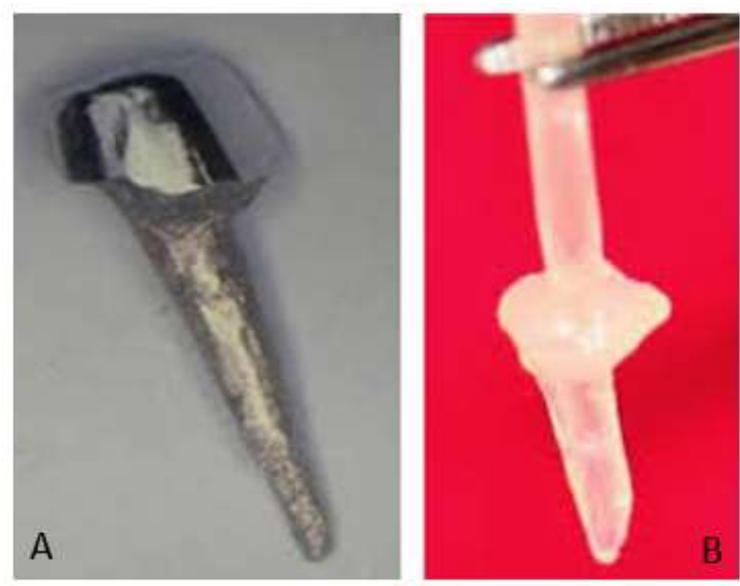

Fuente: Vidalón-Pinto \& Huertas-Mogollón, (2017).

La alternativa más aceptada es el uso de postes de fibra de vidrio (22-57 GPa) (Figura 3B) que han demostrado mayor biocompatibilidad por su módulo de elasticidad similar al de la dentina (42 GPa) (Sorrentino et al., 2016), lo cual produce una distribución de estrés más uniforme y reduce considerablemente el riesgo a la fractura; de igual manera, tiene ventajas a nivel estético y en el tiempo de trabajo (Batista et al., 2020; Marchionatti et al., 2017; Sorrentino et al., 2016).

De acuerdo a la literatura, los premolares maxilares restaurados con postes de fibra de vidrio tienen menor pronóstico debido a la alta incidencia a la fractura vertical asociada al diámetro mesiodistal del conducto radicular (Sorrentino et al., 2016) y a las fuerzas laterales, que son mayores que en los molares. Sin embargo, existe controversia con ciertos estudios donde se menciona que los premolares tienen un rango de sobrevida mayor al de los molares, relacionado con la distancia a la articulación temporomandibular, ya que los molares se encuentran próximos al fulcrum y consecuentemente, expuestos a mayor carga oclusal (Sorrentino et al., 2016; Aquilino \& Caplan, 2002; Chan et al., 1999; Hiatt, 1973; Lynch \& McConnell, 2002; Suksaphar et al., 2018; Tan et al., 2006).

No existen estudios concluyentes que apoyen o rechacen el uso de postes. Empero, Naumann et al., (2018) manifiestan que en la mayoría de estudios clínicos, el poste no ofrece un efecto positivo, sino por el contrario, al implicar la desobturación del conducto radicular, provoca el desgaste interno de tejido dentinario.

Las principales complicaciones que presentan los DTE con postes de fibra y restauraciones de recubrimiento total son: 
- Desadaptación del poste.

- Pérdida de la retención de las coronas.

- Desajuste marginal.

- Fracturas parciales de las coronas o de la prótesis fija plural (Sorrentino et al., 2016)

- Falla en la interfaz adhesiva (Batista et al., 2020)

Circunstancias que se atribuyen a factores como: la cantidad de tejido dental residual, el esquema oclusal, estado periodontal, hábitos, presencia o ausencia de efecto férula, calidad de la adhesión (Sorrentino et al., 2016), ausencia de contactos interproximales, tipo y posición de diente y su relación con el antagonista (Batista et al., 2020).

\subsubsection{DTE con 0 paredes remanentes.}

En aquellas piezas dentarias con pérdida de estructura que sobrepasa el límite amelocementario, se requiere ejecutar procedimientos previos a su restauración como alargamiento de corona o extrusión ortodóntica (Zarow et al., 2018); siendo la primera, la mejor elección para molares y la segunda para premolares (Abdulrazzak et al., 2014; Juloski et al., 2014), para ambos casos se recomienda que el tratamiento sea de al menos $1 \mathrm{~mm}$ (Juloski et al., 2014).

En búsqueda de métodos menos invasivos y que no requieran emplear mayor tiempo de trabajo, se han implementado nuevas técnicas, descritas en los siguientes apartados.

\subsubsection{Elevación de margen profundo}

Consiste en reubicar el margen cervical de una preparación subgingival a una supragingival con el propósito de conseguir un adecuado aislamiento absoluto de la pieza dentaria tras la remoción del tejido carioso, lo que origina la correcta adhesión de varias capas de composite en el margen profundo; creando una nueva restauración marginal, localizada a un nivel más coronal (Sarfati \& Tirlet, 2018); para ello se emplean composites de alta carga. (Bresser et al., 2019)

Para este fin, se manejan resinas fluidas tipo Bulk Fill, indicadas para cavidades clase I y clase II de Black, que se pueden utilizar en incrementos de 4-5 mm con la técnica de monobloque o una sola capa (Corral Núñez et al., 2015). Estudios realizados han demostrado que, al presentar mayor fluidez, mejora su adaptación, y su reducido estrés de polimerización, le otorga la propiedad más requerida, la resistencia a la fractura (Corral Núñez et al., 2015; Rosatto et al., 2015).

La técnica de elevación del margen profundo resulta beneficiosa al ser mínimamente invasiva manteniendo el periodonto intacto, además de que permite al profesional trabajar de manera más segura, en un campo limpio, seco y con un acceso competente, ideal para todo tratamiento restaurador. Un reporte clínico con 12 años de seguimiento, en el que se realizaron inlays y onlays de composite y disilicato de litio en premolares y molares, los cuales se sometieron a este proceso previamente, señalan una tasa de supervivencia entre el 94-96\% (Bresser et al., 2019).

Sarfati \& Tirlet, (2018) realizaron un ensayo clínico controlado donde se compararon los procedimientos de alargamiento de corona y de elevación de margen en dientes posteriores, cuyos resultados reflejan una mejor aceptación de la segunda técnica señalada 180 días después, puesto que la pérdida de inserción clínica fue menor con respecto a la primera. No obstante, otros aspectos como: profundidad de sondaje, índice de placa y profundidad de la bolsa periodontal fueron semejantes en ambos grupos (Oppermann et al., 2016). De modo que es una excelente opción cuando se trata de ser más conservador y preservar tejido. 


\subsubsection{Endocoronas}

Una alternativa mínimamente invasiva que emplea la cámara pulpar para incrementar la retención mecánica de la corona (Figura 4). Se utiliza en piezas con extensiva pérdida de tejido dentario, que posean $1.5 \mathrm{~mm}$ a $2 \mathrm{~mm}$ de altura desde la unión amelocementaria al margen de la preparación dental y $1 \mathrm{~mm}$ mínimo de ancho de paredes remanentes (Al-Dabbagh, 2020; Schwartz \& Robbins, 2004), siendo la razón por la que tienen mejor pronóstico en el sector posterior. Están indicadas en casos de piezas posteriores con raíces cortas, obliteradas, dilaceradas y frágiles, conductos radiculares ensanchados excesivamente, longitud inadecuada de la corona y espacio interoclusal deficiente (Pissis, 1995).

Figura 4: Representación esquemática de la endocorona.

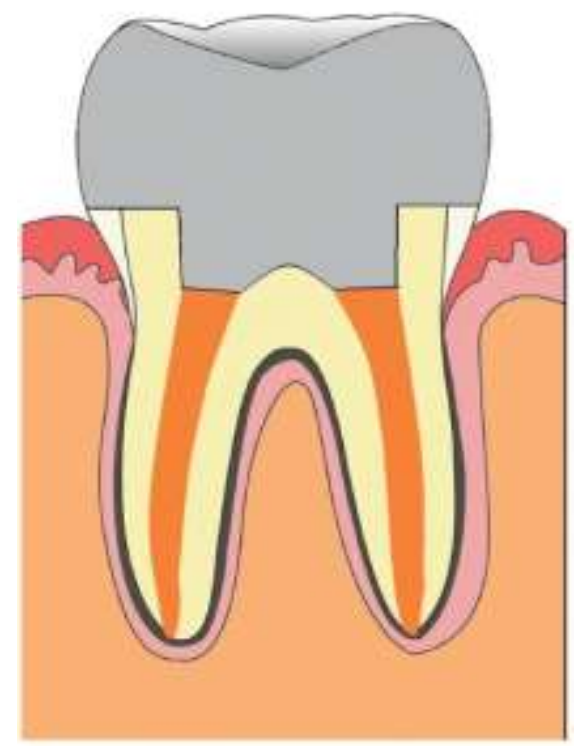

Fuente: Govare \& Contrepois (2019).

Estas suelen ser fabricadas con materiales de bajo módulo de elasticidad, similar al de la dentina ya que ayuda a distribuir las fuerzas a lo largo de la superficie y mejora la resistencia a la fractura (Al-Dabbagh, 2020). Govare y Contrepois, (2019) manifiestan que en la mayoría de estudios clínicos se ha utilizado cerámica feldespática; pero, existen otros materiales como resina con nanopartículas y disilicato de litio que han demostrado mayores ventajas en su fabricación.

Tzimas et al., (2018) reportan éxito a corto plazo y concluyen que existen cuatro factores para el éxito y la longevidad de las endocoronas, como son: preparación correcta del diente, elección precisa del material de restauración, del sistema adhesivo, y una apropiada selección del caso. Al-Dabbagh, (2020) informa que la resistencia a la fractura de las endocoronas es superior o similar a la de las coronas convencionales en dientes posteriores, pues en los estudios evaluados en su revisión se observan tasas de sobrevida mayores al $90 \%$ a corto y largo plazo.

Por ello, se estima que este tipo de restauraciones constituyen una alternativa prometedora para los molares tratados endodónticamente y con gran pérdida de estructura; aunque para premolares sugieren la necesidad de más estudios, especialmente ensayos clínicos que permitan corroborar los resultados obtenidos en estudios in vitro, dado que la tasa de supervivencia en molares es mayor al 90\% en un periodo de 6 meses a 10 años, en relación a los premolares, cuya sobrevida se halla en un rango entre 68\% a 75\% en 55 meses a 10 años. (Govare \& Contrepois, 2019). 


\subsubsection{Técnica de endorestauración}

Consiste en el uso de fibras adheribles de refuerzo (Ribbond) que favorecen a la formación de un bloque sólido en conjunto a composites colocados dentro del complejo intrarradicular, lo cual podría brindar mayor resistencia y estética, ya que la luz refleja de forma semejante a un diente natural. También proporciona la retención necesaria para la restauración definitiva, por consiguiente es crucial que existan $2 \mathrm{~mm}$ de estructura dentaria (Ribbond Inc, n.d.; Iñ́guez, 2000).

Estas fibras se adaptan a la forma del conducto radicular y permiten eliminar la concentración de tensión asociado a los postes preformados, resultando conveniente en casos de retratamientos de conducto o cuando las raíces son inmaduras, lo que predispone a la fractura radicular (Ribbond Inc, n.d.).

A pesar de encontrarse varios años dentro del mercado, no se han presentado estudios que faciliten documentar el éxito de esta técnica a largo plazo, por lo que se requieren más ensayos clínicos in vivo e in vitro para su efecto.

\subsubsection{DTE con pronóstico desfavorable}

Corresponde a la clase 4 de Zarow, en la cual se indica la extracción del DTE, esto puede suceder por dos razones: por la complejidad del tratamiento a realizar (Zarow et al., 2018) o por el lapso de tiempo en el que se coloca la restauración coronal, dando un 25\% más de probabilidad de extraer la pieza si la restauración es efectuada entre 15-59 días posteriores al tratamiento endodóntico y un 73\% luego de los 60 días (Sadaf, 2020).

\section{Conclusiones}

En conclusión, la cantidad de estructura coronal remanente de los dientes tratados endodónticamente es la que determinará el éxito y el tipo de restauración a emplear. Es trascendental conocer la anatomía, las técnicas, procedimientos y biomateriales que surgen día a día en el campo de la odontología, porque permiten generar mejores resultados, confiriendo tratamientos menos invasivos y en un tiempo reducido; lo que constituye una gran ventaja tanto para el profesional como para la persona quien recibe la atención.

Es indispensable destacar la importancia del manejo del paciente entre las especialidades de Endodoncia y Rehabilitación Oral para proporcionar un tratamiento acertado y exitoso a largo plazo.

Por último, cabe mencionar que existe muy poca literatura disponible y actualizada sobre restauraciones directas e indirectas para dientes tratados endodónticamente, sobre todo aquellas de cobertura parcial; por lo tanto, sería conveniente que se realicen más estudios al respecto.

\section{Agradecimientos}

Nuestro extensivo agradecimiento a nuestra tutora Od. Esp.Yulissa Abad por ser una guía durante todo este trabajo y proporcionarnos las fotografías de un caso que fue llevado a cabo en conjunto con la Od. Esp. Lorena Carpio, a quien agradecemos también por su colaboración; les deseamos éxito en sus labores.

\section{Referencias}

Abdulrazzak, S. S., Sulaiman, E., Atiya, B. K., \& Jamaludin, M. (2014). Effect of ferrule height and glass fibre post length on fracture resistance and failure mode of endodontically treated teeth. Australian Endodontic Journal, 40(2), 81-86. https://doi.org/10.1111/aej.12042

Afrashtehfar, K. I., Ahmadi, M., Emami, E., Abi-Nader, S., \& Tamimi, F. (2017). Failure of single-unit restorations on root filled posterior teeth: a systematic review. International Endodontic Journal, 50(10), 951-966. https://doi.org/10.1111/iej.12723

Aguero, P., Paredes, G., \& Alayo, C. (2017). Evolución del poste muñón en Odontología Evolution of intraradicular posts in. Odontologia SanMarquina, 20(2), 75-78. 
Al-Dabbagh, R. A. (2020). Survival and success of endocrowns: A systematic review and meta-analysis. Journal of Prosthetic Dentistry, 1-9. https://doi.org/10.1016/j.prosdent.2020.01.011

Aquilino, S. A., \& Caplan, D. J. (2002). Relationship between crown placement and the survival of endodontically treated teeth. The Journal of Prosthetic Dentistry, 87(3), 256-263. https://doi.org/10.1067/mpr.2002.122014

Atlas, A., Grandini, S., \& Martignoni, M. (2019). Evidence-based treatment planning for the restoration of endodontically treated single teeth: importance of coronal seal, post vs no post, and indirect vs direct restoration. Quintessence International (Berlin, Germany: 1985), 50(10), 772-781. https://doi.org/10.3290/j.qi.a43235

Batista, V. E. de S., Bitencourt, S. B., Bastos, N. A., Pellizzer, E. P., Goiato, M. C., \& dos Santos, D. M. (2020). Influence of the ferrule effect on the failure of fiber-reinforced composite post-and-core restorations: A systematic review and meta-analysis. Journal of Prosthetic Dentistry, 123(2), 239-245. https://doi.org/10.1016/j.prosdent.2019.01.004

Beier, U. S., Kapferer, I., Burtscher, D., Giesinger, J. M., \& Dumfahrt, H. (2012). Clinical performance of all-ceramic inlay and onlay restorations in posterior teeth. The International Journal of Prosthodontics, 25(4), 395-402. http://www.ncbi.nlm.nih.gov/pubmed/22720292

Bitter, K., Noetzel, J., Stamm, O., Vaudt, J., Meyer-Lueckel, H., Neumann, K., \& Kielbassa, A. M. (2009). Randomized Clinical Trial Comparing the Effects of Post Placement on Failure Rate of Postendodontic Restorations: Preliminary Results of a Mean Period of 32 Months. Journal of Endodontics, 35(11), 14771482. https://doi.org/10.1016/j.joen.2009.07.026

Bresser, R. A., Gerdolle, D., van den Heijkant, I. A., Sluiter-Pouwels, L. M. A., Cune, M. S., \& Gresnigt, M. M. M. (2019). Up to 12 years clinical evaluation of 197 partial indirect restorations with deep margin elevation in the posterior region. Journal of Dentistry, 91(November), 103227. https://doi.org/10.1016/j.jdent.2019.103227

Cagidiaco, M. C., Radovic, I., \& Simonetti, M. (2007). Clinical Performance of Fiber Post Restorations in Endodontically Treated Teeth : 2-Year Results. 20(3), 293-298.

Chan, C.-P., Lin, C.-P., Tseng, S.-C., \& Jeng, J.-H. (1999). Vertical root fracture in endodontically versus nonendodontically treated teeth A survey of 315 cases in Chinese patients. Oral Surgery, Oral Medicine, Oral Pathology, Oral Radiology, and Endodontology, 87(4), 504-507. https://doi.org/10.1016/S10792104(99)70252-0

Corral Núñez, C., Vildósola Grez, P., Bersezio Miranda, C., Alves Dos Campos, E., \& Fernández Godoy, E. (2015). Revisión del estado actual de resinas compuestas bulk-fill. Revista Facultad de Odontología, 27(1). https://doi.org/10.17533/udea.rfo.v27n1a9

Dammaschke, T., Nykiel, K., Sagheri, D., \& Schäfer, E. (2013). Influence of coronal restorations on the fracture resistance of root canal-treated premolar and molar teeth: A retrospective study. Australian Endodontic Journal, 39(2), 48-56. https://doi.org/10.1111/aej.12002

Ferrari, M., Ferrari Cagidiaco, E., Goracci, C., Sorrentino, R., Zarone, F., Grandini, S., \& Joda, T. (2019). Posterior partial crowns out of lithium disilicate (LS2) with or without posts: A randomized controlled prospective clinical trial with a 3-year follow up. Journal of Dentistry, 83(December 2018), 12-17. https://doi.org/10.1016/j.jdent.2019.01.004

Ferraris, F. (2017). Posterior indirect adhesive restorations (PIAR): preparation designs and adhesthetics clinical protocol. The International Journal of Esthetic Dentistry, 12(4), 482-502.

Govare, N., \& Contrepois, M. (2019). Endocrowns: A systematic review. Journal of Prosthetic Dentistry, 123(3), 411-418.e9. https://doi.org/10.1016/j.prosdent.2019.04.009

Hiatt, W. H. (1973). Incomplete Crown-Root Fracture in Pulpal-Periodontal Disease. Journal of Periodontology, 44(6), 369-379. https://doi.org/10.1902/jop.1973.44.6.369

Iñíguez, I. (2000). Odontología restaurativa directa. Usos de RIBBOND para restaurar dientes tratados endodónticamente. Revista de La Asociación Dental Mexicana, 57(2), 54-58.

Jirathanyanatt, T., Suksaphar, W., Banomyong, D., \& Ngoenwiwatkul, Y. (2019). Endodontically treated posterior teeth restored with or without crown restorations: A 5-year retrospective study of survival rates from fracture. Journal of Investigative and Clinical Dentistry, 10(4), e12426. https://doi.org/10.1111/jicd.12426

Juloski, J., Apicella, D., \& Ferrari, M. (2014). The effect of ferrule height on stress distribution within a tooth restored with fibre posts and ceramic crown: A finite element analysis. Dental Materials, 30(12), 1304-1315. https://doi.org/10.1016/j.dental.2014.09.004

Lynch, C. D., \& McConnell, R. J. (2002). The cracked tooth syndrome. Journal (Canadian Dental Association), 68(8), 470-475. https://doi.org/12323102 Magne, P., \& Spreafico, R. C. (2012). Deep Margin Elevation : Am J Esthet Dent, 2(2), 86-96.

Mannocci, F., Bertelli, E., Sherriff, M., Watson, T. F., \& Pitt Ford, T. R. (2009). Three-year clinical comparison of survival of endodontically treated teeth restored with either full cast coverage or with direct composite restoration. International Endodontic Journal, 42(5), 401-405. https://doi.org/10.1111/j.13652591.2009.01559.x

Marchionatti, A. M. E., Wandscher, V. F., Rippe, M. P., Kaizer, O. B., \& Valandro, L. F. (2017). Clinical performance and failure modes of pulpless teeth restored with posts: a systematic review. Brazilian Oral Research, 31, e64. https://doi.org/10.1590/1807-3107BOR-2017.vol31.0064

Monardes, H., Lolas, C., Aravena, J., González, H., \& Abarca, J. (2016). Evaluación del tratamiento endodóntico y su relación con el tipo y la calidad de la restauración definitiva. Revista Clínica de Periodoncia, Implantología y Rehabilitación Oral, 9(2), 108-113. https://doi.org/10.1016/j.piro.2016.03.004

Naumann, M., Schmitter, M., Frankenberger, R., \& Krastl, G. (2017). "Ferrule Comes First. Post Is Second!” Fake News and Alternative Facts? A Systematic Review. Journal of Endodontics, 44(2), 212-219. https://doi.org/10.1016/j.joen.2017.09.020 
Naumann, M., Schmitter, M., \& Krastl, G. (2018). Postendodontic restoration: Endodontic post-and-core or no post at all? The Journal of Adhesive Dentistry, 20(1), 19-24. https://doi.org/10.3290/j.jad.a39961

Oppermann, R., Gomes, S., Cavagni, J., Cayana, E., \& Conceição, E. (2016). Response to Proximal Restorations Placed Either Subgingivally or Following Crown Lengthening in Patients with No History of Periodontal Disease. The International Journal of Periodontics \& Restorative Dentistry, 36(1), 117-124. https://doi.org/10.11607/prd.2015

Peroz, I., Blankenstein, F., Lange, K.-P., \& Naumann, M. (2005). Restoring endodontically treated teeth with posts and cores--a review. Quintessence International (Berlin, Germany : 1985), 36(9), 737-746. http://www.ncbi.nlm.nih.gov/pubmed/16163877

Pissis, P. (1995). Fabrication of a metal-free ceramic restoration utilizing the monobloc technique. Practical Periodontics and Aesthetic Dentistry : PPAD, 7(5), 83-94. http://www.ncbi.nlm.nih.gov/pubmed/7548896

Ribbond Inc. (n.d.). Manual Ribbond: cinta de refuerzo adhesivo. https://www.sasexclusivasdentales.es/img/cms/Manuales/Manual Ribbond.pdf

Rosatto, C. M. P., Bicalho, A. A., Veríssimo, C., Bragança, G. F., Rodrigues, M. P., Tantbirojn, D., Versluis, A., \& Soares, C. J. (2015). Mechanical properties, shrinkage stress, cuspal strain and fracture resistance of molars restored with bulk-fill composites and incremental filling technique. Journal of Dentistry, 43(12), 1519-1528. https://doi.org/10.1016/j.jdent.2015.09.007

Sadaf, D. (2020). Survival rates of endodontically treated teeth after placement of definitive coronal restoration: 8-year retrospective study. Therapeutics and Clinical Risk Management, 16, 125-131. https://doi.org/10.2147/TCRM.S223233

Sarfati, A., \& Tirlet, G. (2018). Deep margin elevation versus crown lengthening: biologic width revisited. The International Journal of Esthetic Dentistry, $13(3), 334-356$.

Sarkis-Onofre, R., Fergusson, D., Cenci, M. S., Moher, D., \& Pereira-Cenci, T. (2017). Performance of Post-retained Single Crowns: A Systematic Review of Related Risk Factors. Journal of Endodontics, 43(2), 175-183. https://doi.org/10.1016/j.joen.2016.10.025

Schwartz, R., \& Robbins, J. (2004). Post Placement and Restoration of Endodontically Treated Teeth: A Literature Review. Journal of Endodontics, 30(5), 289-301. https://doi.org/10.1097/00004770-200405000-00001

Shu, X., Mai, Q.-Q., Blatz, M., Price, R., Wang, X.-D., \& Zhao, K. (2018). Direct and Indirect Restorations for Endodontically Treated Teeth: A Systematic Review and Meta-analysis, IAAD 2017 Consensus Conference Paper. The Journal of Adhesive Dentistry, 20(3), 183-194. https://doi.org/10.3290/j.jad.a40762

Sorrentino, R., Di Mauro, M. I., Ferrari, M., Leone, R., \& Zarone, F. (2016). Complications of endodontically treated teeth restored with fiber posts and single crowns or fixed dental prostheses-a systematic review. Clinical Oral Investigations, 20(7), 1449-1457. https://doi.org/10.1007/s00784-016-1919-8

Suksaphar, W., Banomyong, D., Jirathanyanatt, T., \& Ngoenwiwatkul, Y. (2018). Survival Rates from Fracture of Endodontically Treated Premolars Restored with Full-coverage Crowns or Direct Resin Composite Restorations: A Retrospective Study. Journal of Endodontics, 44(2), 233-238. https://doi.org/10.1016/j.joen.2017.09.013

Tan, L., Chen, N. N., Poon, C. Y., \& Wong, H. B. (2006). Survival of root filled cracked teeth in a tertiary institution. International Endodontic Journal, 39(11), 886-889. https://doi.org/10.1111/j.1365-2591.2006.01165.x

Tzimas, K., Tsiafitsa, M., Gerasimou, P., \& Tsitrou, E. (2018). Endocrown restorations for extensively damaged posterior teeth: clinical performance of three cases. Restorative Dentistry \& Endodontics, 43(4), 1-9. https://doi.org/10.5395/rde.2018.43.e38

Vallejo Labrada, M., \& Maya Cerón, C. X. (2015). Influencia de la calidad de restauración coronal en el pronóstico de dientes tratados endodónticamente. Revista Cubana de Estomatologia, 52(1), 47-62.

Victoria, D. (2019). Coronal restoration in root-filled and non root-filled teeth. U. M. Facultad de Odontología.

Vidalón-Pinto, M., \& Huertas-Mogollón, G. (2017). Rehabilitación del diente tratado endodonticamente: poste colado versus poste de fibra de vidrio. Revista Cientifica Unviersidad Odontológica Dominicana, 5(1), 660-667.

Zarow, M., Ramírez-Sebastià, A., Paolone, G., de Ribot Porta, J., Mora, J., Espona, J., Durán-Sindreu, F., \& Roig, M. (2018). A new classification system for the restoration of root filled teeth. International Endodontic Journal, 51(3), 318-334. https://doi.org/10.1111/iej.12847 